\title{
Dorsal Spinal Ligamentum Flavum Thickening: A Magnetic Resonance Imaging Study
}

\author{
Amarnath Chelladurai, Suhasini Balasubramaniam, Sarenya Preyah Anbazhagan, \\ Sathyan Gnanasihamani, Sukumar Ramaswami
}

Department of Radiodiagnosis, Stanley Medical College, Chennai, India

\begin{abstract}
Study Design: A retrospective radiological study of the ligamentum flavum (LF).
Purpose: We determined the relationship of dorsal spinal LF thickening with age and sex using magnetic resonance imaging (MRI). We also determined whether LF thickening has a predominant tendency to occur at a specific dorsal level and on a specific side.

Overview of Literature: Many researchers have studied LF thickness at dorsal levels in patients with compressive myelopathy. However, there is a dearth of literature pertaining to the study of dorsal LF thickness in patients without myelopathy.

Methods: LF thickness was measured at dorsal levels from T1 to T12 on both sides using MRI in 100 individuals. The patients were divided into three groups based on age: 20 to 40,41 to 60, and $>60$ years. On axial T2-weighted imaging at the mid-disc level, LF thickness was measured perpendicular to the lamina border, either at half the length of $L F$ or at maximum thickness, whichever was greater.

Results: We found that LF thickness does not increase significantly with increasing age and there was no significant disparity in LF thickness between the sides and sexes. We also found that there was a significant increase in LF thickness at the T10-T11 level (mean value, $3.27 \pm 0.94 \mathrm{~mm}$ ).

Conclusions: LF thickness does not appear to have any side/sex dominance. LF thickening has a predominant tendency to occur specifically at the T10-T11 level. This may be due to maximum tensile strength and mobility at this level. Because there is an increased tendency for LF thickening at the T10-T11 level, this may be used as a reference point for counting the vertebral levels.
\end{abstract}

Keywords: Ligamentum flavum thickening; T10-T11; Dorsal levels; Age

\section{Introduction}

Many ligaments have a major role in reinforcement and mechanical support of the joints between vertebrae; one of them is the ligamentum flavum (LF). LF or yellow ligament is a ligament of the spine that connects the laminae of adjacent vertebrae from the $\mathrm{C} 2$ vertebra to the first seg- ment of the sacrum. LF contains longitudinally arranged connective tissue fibers that are predominantly elastic in nature. These ligaments extend from the anterior/inferior aspect of the lamina above a disc space to the posterior/ superior aspect of the lamina below the disc space [1].

Degeneration with thickening and calcification of LF can cause foraminal narrowing, spinal canal stenosis, and

Received Apr 3, 2017; Revised Apr 14, 2017; Accepted Apr 18, 2017

Corresponding author: Suhasini Balasubramaniam

Department of Radiodiagnosis, Stanley Medical College, Chennai Tamilnadu, IN 600001, India

Tel: +917401123474, E-mail: drsuhasmd@gmail.com 
nerve root compression at the lumbar level [2]. LF thickening is an important cause of radiculopathy in lumbar degenerative disease. Back pain has become a significant healthcare concern in middle-aged and elderly individuals. It affects the quality of life significantly and usually is diagnosed late due to its indolent course. Hence, early diagnosis and initiation of treatment can improve the patient's outcome [3].

Either an increase in LF thickness or a posterior disc protrusion may cause spinal canal stenosis and compression of the nerve roots. The mechanism of LF thickening remains controversial. Thickening and hypertrophy of the ligament probably results from injury. Normally, LF comprises yellow elastic fibers, which are responsible for its elasticity [4]. LF is the main apposition to flexion loading of the spine, and it can be injured with excessive spinal flexion. Unfortunately, there is little or no regenerative capacity in the elastic tissue of LF; thus, it is replaced by a dense connective tissue cicatrix. This repair mechanism with scar tissue formation and with further enlargement of the ligament may result in compression of the nerve roots [5].

Most studies investigating LF thickness have examined and assessed the lumbar region. Few studies and data in the literature pertain to changes in LF thickness at the dorsal levels.

We aimed to provide details of LF thickness at the dorsal spinal levels and determine its relationship with age, sex, and sides.

\section{Materials and Methods}

Institutional Ethical Committee approval was obtained (Reg. no-Ecr/131/inst/TN/2013) before the start of this 2-year (June 2015-2017) retrospective study of 100 patients (58 males, 42 females; age $>20$ years) who presented for complaints other than back pain. We divided the patients into three groups based on age: 20 to 40,41 to 60 ,

Table 1. Distribution of study group as per age in years

\begin{tabular}{lc} 
Age (yr) & Frequency $(\%)$ \\
$20-40$ & 44 \\
$41-60$ & 34 \\
$>60$ & 22 \\
Total & 100 \\
\hline
\end{tabular}

and $>60$ years (Table 1 ). Patients aged $<20$ years or with severe degenerative disc disease, history of previous spine surgery and radiotherapy, congenital abnormality, spinal deformities, pacemakers, metallic implants, and joint replacements were excluded. LF thickness was measured by a radiologist experienced in spine magnetic resonance imaging (MRI).

\section{Magnetic resonance imaging protocol}

Imaging was performed on a 48-channel SIEMENS AERA

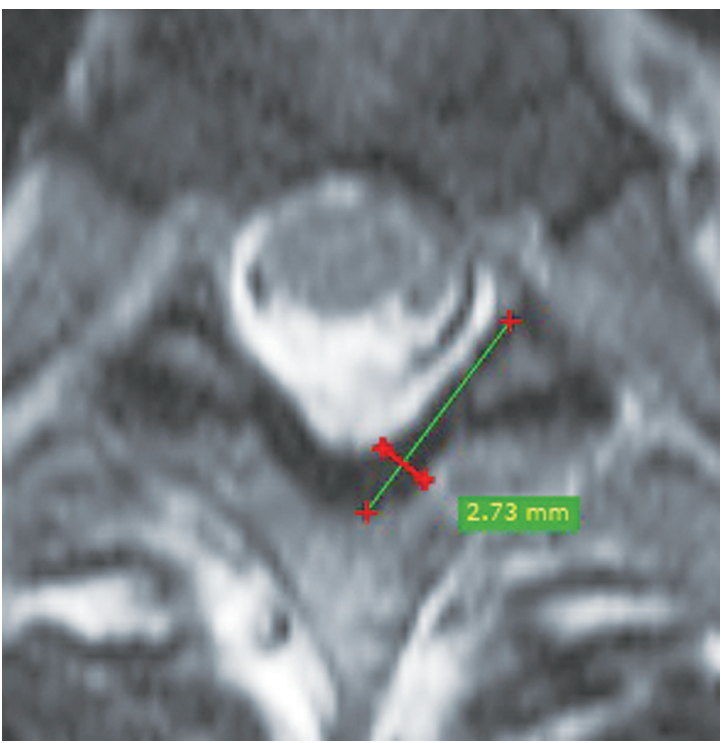

Fig. 1. Axial T2-weighted imaging showing ligamentum flavum measurements.

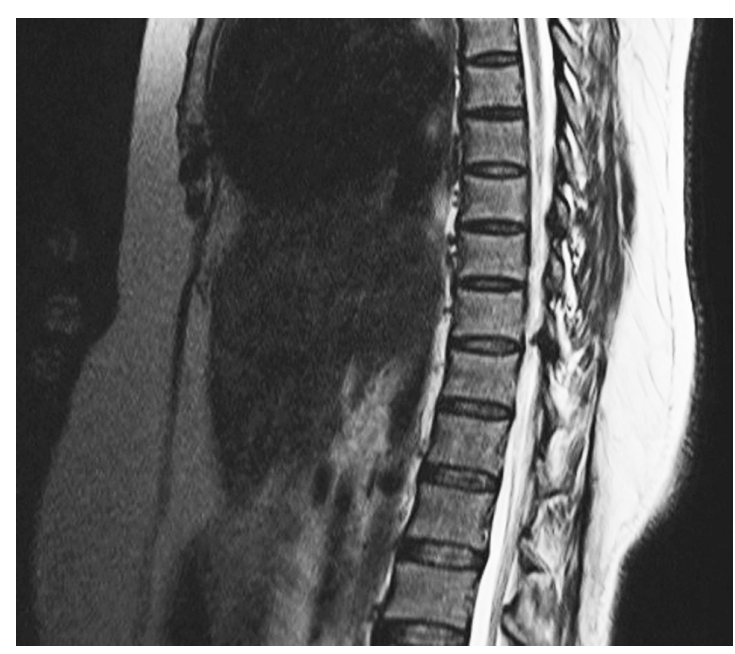

Fig. 2. Sagittal T2 magnetic resonance image showing ligamentum flavum thickening at T10-T11 level. 
system 1.5T (Siemens Medical Solutions, Erlangen, Germany) with the standard protocol. In every patient, MRI of the whole spine was performed in the axial and sagittal planes as per our institutional protocol. A mid sagittal T2weighted image (T2WI) of the whole spine was used to count the spinal level of intervertebral spaces, after which the LF thickness was measured on axial T2WIs at dorsal spinal levels, at the mid-disc level perpendicular to the lamina border, either at half the length of LF or at maxi-

Table 2. Comparison of mean LF thickness between different age groups

\begin{tabular}{lccc}
$\begin{array}{l}\text { LF thickness } \\
\text { vs. age }\end{array}$ & $\begin{array}{c}21-40 \mathrm{yr} \\
\text { group }\end{array}$ & $\begin{array}{c}40-60 \mathrm{yr} \\
\text { group }\end{array}$ & $\begin{array}{c}>60 \mathrm{yr} \\
\text { group }\end{array}$ \\
Age $(\mathrm{yr})$ & $31.39 \pm 4.85$ & $49.15 \pm 5.48$ & $66.50 \pm 3.76$ \\
T1-T2 (mm) & $1.84 \pm 0.62$ & $1.91 \pm 0.48$ & $1.87 \pm 0.60$ \\
\hline T2-T3 (mm) & $1.95 \pm 0.46$ & $2.03 \pm 0.58$ & $1.92 \pm 0.49$ \\
T3-T4 (mm) & $2.25 \pm 0.51$ & $2.35 \pm 0.53$ & $2.23 \pm 0.48$ \\
T4-T5 (mm) & $2.62 \pm 0.53$ & $2.71 \pm 0.60$ & $2.52 \pm 0.51$ \\
T5-T6 (mm) & $2.75 \pm 0.71$ & $2.88 \pm 0.62$ & $2.81 \pm 0.64$ \\
\hline T6-T7 (mm) & $2.91 \pm 0.56$ & $3.09 \pm 0.65$ & $2.99 \pm 0.59$ \\
T7-T8 (mm) & $3.00 \pm 0.89$ & $2.79 \pm 0.83$ & $3.09 \pm 0.66$ \\
\hline T8-T9 (mm) & $2.92 \pm 0.75$ & $2.81 \pm 0.90$ & $2.90 \pm 0.63$ \\
\hline T9-T10 (mm) & $2.75 \pm 0.76$ & $2.93 \pm 0.96$ & $2.87 \pm 0.57$ \\
\hline T10-T11 (mm) & $3.07 \pm 0.93$ & $3.18 \pm 0.95$ & $3.19 \pm 0.71$ \\
\hline T11-T12 (mm) & $2.64 \pm 0.74$ & $2.65 \pm 0.73$ & $2.48 \pm 0.57$ \\
\hline
\end{tabular}

Values are presented as mean \pm standard deviation.

$\mathrm{LF}$, ligamentum flavum.

Table 3. Correlation between LF thickness and age

\begin{tabular}{lcc} 
Parameter & Mean \pm standard deviation & Pearson $r$ \\
\hline Age $(\mathrm{yr})$ & $45.15 \pm 14.64$ & - \\
\hline T1-T2 $(\mathrm{mm})$ & $1.87 \pm 0.57$ & -0.02 \\
\hline T2-T3 $(\mathrm{mm})$ & $1.97 \pm 0.51$ & 0.04 \\
T3-T4 $(\mathrm{mm})$ & $2.28 \pm 0.51$ & 0.07 \\
\hline T4-T5 $(\mathrm{mm})$ & $2.63 \pm 0.56$ & -0.07 \\
T5-T6 $(\mathrm{mm})$ & $2.81 \pm 0.66$ & 0.03 \\
\hline T6-T7 $(\mathrm{mm})$ & $2.99 \pm 0.60$ & 0.05 \\
$\mathrm{~T} 7-\mathrm{T} 8(\mathrm{~mm})$ & $2.95 \pm 0.83$ & 0.07 \\
\hline T8-T9 $(\mathrm{mm})$ & $2.88 \pm 0.78$ & 0.05 \\
T9-T10 $(\mathrm{mm})$ & $2.84 \pm 0.80$ & 0.01 \\
\hline T10-T11 $(\mathrm{mm})$ & $3.13 \pm 0.89$ & -0.04 \\
\hline T11-T12 $(\mathrm{mm})$ & $2.61 \pm 0.70$ & 0.06 \\
\hline
\end{tabular}

LF, ligamentum flavum. mum thickness, whichever was greater (Fig. 1). Increased LF thickening at the T10-T11 level is shown in Fig. 2.

The mean LF thickness in three age groups (20 to 40, 41 to 60 , and $>60$ years) was analyzed in relation to sides and sex. Mean values of LF thickness were analyzed for the entire dorsal levels.

\section{Data analysis}

Descriptive statistics were analyzed for all data, and suit-

Table 4. Comparison of mean LF thickness between male and female group

\begin{tabular}{lcc}
\hline LF thickness vs. sex & Male group & Female group \\
\hline Age $(\mathrm{yr})$ & $44.28 \pm 14.33$ & $46.45 \pm 15.00$ \\
\hline T1-T2 $(\mathrm{mm})$ & $1.92 \pm 0.65$ & $1.79 \pm 0.42$ \\
\hline T2-T3 $(\mathrm{mm})$ & $1.98 \pm 0.55$ & $1.97 \pm 0.44$ \\
\hline T3-T4 $(\mathrm{mm})$ & $2.25 \pm 0.54$ & $2.34 \pm 0.47$ \\
\hline T4-T5 $(\mathrm{mm})$ & $2.62 \pm 0.60$ & $2.64 \pm 0.49$ \\
\hline T5-T6 $(\mathrm{mm})$ & $2.85 \pm 0.68$ & $2.75 \pm 0.64$ \\
\hline T6-T7 $(\mathrm{mm})$ & $3.03 \pm 0.61$ & $2.93 \pm 0.59$ \\
T7-T8 $(\mathrm{mm})$ & $2.94 \pm 0.82$ & $2.96 \pm 0.84$ \\
\hline T8-T9 $(\mathrm{mm})$ & $2.91 \pm 0.81$ & $2.83 \pm 0.72$ \\
T9-T10 $(\mathrm{mm})$ & $2.92 \pm 0.88$ & $2.72 \pm 0.65$ \\
T10-T11 $(\mathrm{mm})$ & $3.20 \pm 0.92$ & $3.03 \pm 0.85$ \\
\hline T11-T12 $(\mathrm{mm})$ & $2.64 \pm 0.71$ & $2.57 \pm 0.69$ \\
\hline
\end{tabular}

Values are presented as mean \pm standard deviation.

$\mathrm{LF}$, ligamentum flavum.

Table 5. Comparison of mean LF thickness between right and left side

\begin{tabular}{lcc}
\hline LF thickness vs. sides & Right side & Left side \\
\hline T1-T2 $(\mathrm{mm})$ & $1.98 \pm 0.52$ & $2.05 \pm 0.56$ \\
\hline T2-T3 $(\mathrm{mm})$ & $1.99 \pm 0.47$ & $2.07 \pm 0.53$ \\
\hline T3-T4 $(\mathrm{mm})$ & $2.22 \pm 0.50$ & $2.34 \pm 0.52$ \\
\hline T4-T5 $(\mathrm{mm})$ & $2.64 \pm 0.57$ & $2.62 \pm 0.54$ \\
\hline T5-T6 $(\mathrm{mm})$ & $2.79 \pm 0.65$ & $2.83 \pm 0.68$ \\
\hline T6-T7 $(\mathrm{mm})$ & $2.97 \pm 0.55$ & $3.01 \pm 0.65$ \\
T7-T8 $(\mathrm{mm})$ & $3.03 \pm 0.83$ & $2.86 \pm 0.82$ \\
T8-T9 $(\mathrm{mm})$ & $3.01 \pm 0.85$ & $2.80 \pm 0.76$ \\
T9-T10 $(\mathrm{mm})$ & $2.84 \pm 0.83$ & $2.84 \pm 0.77$ \\
T10-T11 $(\mathrm{mm})$ & $3.17 \pm 0.90$ & $3.09 \pm 0.89$ \\
T11-T12 $(\mathrm{mm})$ & $2.63 \pm 0.70$ & $2.59 \pm 0.71$ \\
\hline
\end{tabular}

Values are presented as mean \pm standard deviation.

$\mathrm{LF}$, ligamentum flavum. 
Table 6. Mean LF thickness values

\begin{tabular}{|c|c|c|c|c|}
\hline LF thickness (mean values) & $21-40 \mathrm{yr}$ & $41-60 \mathrm{yr}$ & $>60 \mathrm{yr}$ & Mean \\
\hline D1-D7 & $2.39 \pm 0.70$ & $2.50 \pm 0.72$ & $2.39 \pm 0.69$ & $2.43 \pm 0.71$ \\
\hline D7-D12 & $2.88 \pm 0.84$ & $2.86 \pm 0.88$ & $2.94 \pm 0.71$ & $2.89 \pm 0.82$ \\
\hline D1-D12 & $2.61 \pm 0.80$ & $2.67 \pm 0.82$ & $2.64 \pm 0.75$ & $2.64 \pm 0.80$ \\
\hline
\end{tabular}

Values are presented as mean \pm standard deviation.

LF, ligamentum flavum.

Table 7. LF thickness at upper dorsal levels

\begin{tabular}{lc}
$\begin{array}{l}\text { LF thickness at upper } \\
\text { dorsal levels }\end{array}$ & Mean \pm standard deviation \\
T1-T2 (mm) & $1.87 \pm 0.57$ \\
T2-T3 (mm) & $1.97 \pm 0.51$ \\
\hline T3-T4 (mm) & $2.28 \pm 0.51$ \\
T4-T5 (mm) & $2.63 \pm 0.56$ \\
T5-T6 (mm) & $2.81 \pm 0.66$ \\
\hline T6-T7 (mm) & $2.99 \pm 0.60$ \\
\hline
\end{tabular}

LF, ligamentum flavum.

able statistical tests of comparison were performed. Continuous variables were analyzed with the unpaired $t$-test and one-way analysis of variance. Correlation analysis was done using Pearson's $r$ correlation. Statistical significance was set at $p<0.05$. The data were analyzed using SPSS ver. 16.0 software package (SPSS Inc., Chicago, IL, USA).

\section{Results}

Mean LF thickness from T1 to T12 in different age groups is shown in Table 2. There was no significant increase in LF thickness with respect to age. Pearson's correlation revealed no correlation between LF thickness and age (Table 3). There was no significant difference in mean LF thickness between male and female sexes (Table 4) and between the two sides (Table 5). Mean LF thickness at the upper, lower, and entire dorsal levels separately in different age groups was analyzed (Table 6), and there was no significant thickening at any of the upper dorsal levels (T1-T7) (Table 7). However, analysis of mean LF thickness for the entire dorsal levels showed a significant increase $(p<0.001)$ at the T10-T11 level $(3.27 \pm 0.94 \mathrm{~mm})$ (Table 8).

\section{Discussion}

Many investigators have studied LF thickness at dorsal
Table 8. LF thickness at entire dorsal levels

\begin{tabular}{|c|c|}
\hline $\begin{array}{l}\text { LF thickness at dorsal } \\
\text { levels }\end{array}$ & Mean \pm standard deviation \\
\hline $\mathrm{T} 1-\mathrm{T} 2(\mathrm{~mm})$ & $1.87 \pm 0.57$ \\
\hline $\mathrm{T} 2-\mathrm{T} 3$ (mm) & $1.97 \pm 0.51$ \\
\hline T3-T4 (mm) & $2.28 \pm 0.51$ \\
\hline T4-T5 (mm) & $2.63 \pm 0.56$ \\
\hline T5-T6 (mm) & $2.81 \pm 0.66$ \\
\hline $\mathrm{T} 6-\mathrm{T} 7(\mathrm{~mm})$ & $2.99 \pm 0.60$ \\
\hline T7-T8 (mm) & $3.05 \pm 0.87$ \\
\hline T8-T9 (mm) & $3.04 \pm 0.84$ \\
\hline T9-T10 (mm) & $2.93 \pm 0.89$ \\
\hline $\mathrm{T} 10-\mathrm{T} 11(\mathrm{~mm})$ & $3.27 \pm 0.94^{* * *}$ \\
\hline $\mathrm{T} 11-\mathrm{T} 12$ (mm) & $2.70 \pm 0.74$ \\
\hline
\end{tabular}

$\mathrm{LF}$, ligamentum flavum.

***Significant at $p<0.001$.

levels in patients with compressive myelopathy. However, there is a dearth of literature pertaining to the study of dorsal LF thickness in patients without myelopathy. Many hypotheses explain LF thickening, including age-related changes and the gradual replacement of elastin fibers in the ligaments with collagen [6]. Chokshi et al. [7] reported that degenerative diseases caused asymmetrical LF thickening, and Safak et al. [8] found no association between LF thickness and sex or age. We found that LF thickening in the dorsal spine has no correlation with age, and it does not increase with increasing age. There was no significant disparity in LF thickness between the two sides and between male and female sexes.

Among upper thoracic levels from T1 to T6, there was no significant increase in LF thickness at any specific level. However, at lower dorsal levels, there was a significant increase in LF thickness at the T10-T11 level. We presume that this may be due to maximum tensile strength at this level [9]. The lower dorsal spine has greater mobility in flexion and extension [10]. One reason why ossification of 
LF develops more frequently in East Asia is considered to be due to the more frequent squatting position adopted by members of those populations [11]. The predilection sites for ossification of LF in patients with thoracic myelopathy are the lower, high, and mid-thoracic areas in order of frequency. The most frequent site is T10-T11, and it is presumed that this is because these segments receive the maximum tensile force [11].

Calcification and ossification of LF, a frequent cause of compressive myelopathy, mainly affect the lower thoracic levels of the spinal cord, causing progressive spastic paraplegia [12]. Hirabayashi et al. [13] and Park et al. [14] also found that ossification of LF overlying the lower thoracic spine frequently produces myelopathy and the most frequent site of ossification is the T10-T11 level. Because there is increased tendency of LF thickening at the T10T11 level, this may be used as a reference point for counting the vertebral levels.

\section{Conclusions}

LF thickness does not increase with increasing age, and it does not appear to have any side/sex dominance. LF thickening has a predominant tendency to occur specifically at the T10-T11 level, which may be due to maximum tensile strength at this level. Hence, LF thickening at the T10T11 level may be used as a reference point for counting the vertebral levels.

Our study has certain limitations. It did not include patients with compressive myelopathy, and we did not study dorsal spinal LF thickening with respect to disc space narrowing and facet hypertrophy. Low sample size may have influenced the results. Future studies could include assessment of dorsal spinal LF thickness in relation to degenerative disc disease.

\section{Conflict of Interest}

No potential conflict of interest relevant to this article was reported.

\section{References}

1. Sinnatamby CS, Last RJ. Last's anatomy: regional and applied. Edinburgh: Churchill Livingstone/Elsevier; 2011.

2. Liu LM, Song YM, Gong Q. Treatment of lumbar stenosis and root pain resulting from simple hypertrophy of lumbar ligamentum flavum. Zhongguo Xiu Fu Chong Jian Wai Ke Za Zhi 2003;17:50-1.

3. Alvarez JA, Hardy RH Jr. Lumbar spine stenosis: a common cause of back and leg pain. Am Fam Physician 1998;57:1825-34.

4. Kolte VS, Khambatta S, Ambiye MV. Thickness of the ligamentum flavum: correlation with age and its asymmetry-an magnetic resonance imaging study. Asian Spine J 2015;9:245-53.

5. Brown HA. Enlargement of the ligamentum flavum. J Bone Joint Surg Am 1938;20:325-38.

6. Schrader PK, Grob D, Rahn BA, Cordey J, Dvorak J. Histology of the ligamentum flavum in patients with degenerative lumbar spinal stenosis. Eur Spine J 1999;8:323-8.

7. Chokshi FH, Quencer RM, Smoker WR. The "thickened" ligamentum flavum: is it buckling or enlargement? AJNR Am J Neuroradiol 2010;31:1813-6.

8. Safak AA, Is M, Sevinc O, et al. The thickness of the ligamentum flavum in relation to age and gender. Clin Anat 2010;23:79-83.

9. Kosaka H, Sairyo K, Biyani A, et al. Pathomechanism of loss of elasticity and hypertrophy of lumbar ligamentum flavum in elderly patients with lumbar spinal canal stenosis. Spine (Phila Pa 1976) 2007;32:2805-11.

10. Jayakumar PN, Devi BI, Bhat DI, Das BS. Thoracic cord compression due to ossified hypertrophied ligamentum flavum. Neurol India 2002;50:286-9.

11. Ahn DK, Lee S, Moon SH, Boo KH, Chang BK, Lee JI. Ossification of the ligamentum flavum. Asian Spine J 2014;8:89-96.

12. Shiguematsu FY, de Souza EC, Zimmermann AF, Castro GR, Pereira IA, Neves FS. Thoracic myelopathy due to calcification of the ligamentum flavum with hyperproteinorachia and responsive to steroid therapy: case report. Rev Bras Reumatol 2012;52:43846.

13. Hirabayashi H, Ebara S, Takahashi J, et al. Surgery for thoracic myelopathy caused by ossification of the ligamentum flavum. Surg Neurol 2008;69:114-6.

14. Park BC, Min WK, Oh CW, et al. Surgical outcome of thoracic myelopathy secondary to ossification of ligamentum flavum. Joint Bone Spine 2007;74:600-5. 